\title{
Lattice location study of ion implanted Sn and Sn-related defects in Ge
}

\author{
S. Decoster, ${ }^{1}$ S. Cottenier, ${ }^{2,3}$ U. Wahl, ${ }^{4}$ J.G. Correia, ${ }^{4}$ and A. Vantomme ${ }^{1}$ \\ ${ }^{1}$ Instituut voor Kern- en Stralingsfysica and INPAC, KULeuven, BE-3001 Leuven, Belgium* \\ ${ }^{2}$ Instituut voor Kern- en Stralingsfysica and INPAC, K.U.Leuven, BE-3001 Leuven, Belgium \\ ${ }^{3}$ Center for Molecular Modeling, Ghent University, Proeftuinstraat 86, BE-9000 Ghent, Belgium \\ ${ }^{4}$ Instituto Tecnológico e Nuclear, Unidade de Física e Aceleradores, \\ Estrada Nacional 10, apt. 21, 2686-953 Sacavém, Portugal
}

(Dated: December 16, 2009)

\begin{abstract}
In this work, we present a lattice location study of Sn in Ge. From emission channeling experiments, we determined the exact lattice location of ion implanted ${ }^{121} \mathrm{Sn}$ atoms, and compared the results to predictions from density functional calculations. The majority of the Sn atoms are positioned on the substitutional site, as can be expected for an isovalent impurity, while a second significant fraction occupies the six-fold coordinated bond-centered site. Corroborated by ab initio calculations, we attribute this fraction of bond-centered Sn atoms to the Sn-vacancy defect complex in the split-vacancy configuration. Furthermore, we are able to assign specific defect complex geometries to resonances from earlier Mössbauer spectroscopy studies of Sn in Ge.
\end{abstract}

PACS numbers:

\section{INTRODUCTION}

Ge is considered as an important future material to replace $\mathrm{Si}$ in semiconducting applications. Compared to silicon, Ge has a higher free carrier mobility and a lower dopant activation temperature ${ }^{1}$, which makes it an attractive material in future metal-oxide semiconductor field-effect transistors ${ }^{2,3}$. To control the growth of Gebased devices, a thorough understanding of the diffusion properties and mechanism is needed. It is generally accepted that most group III and V dopants (with the exception of B) in Ge diffuse by vacancy mediation. Among all impurities in group IV semiconductors, the diffusion behavior of $\mathrm{Sn}$ is one of the most interesting to investigate: $\mathrm{Sn}$ is an isovalent impurity in Ge, which means that its diffusion properties are likely to be related to Ge self-diffusion. Moreover, the investigation of Sn-related defects in Ge is a technologically important issue since $\mathrm{Sn}_{x} \mathrm{Ge}_{1-x}$ is known as a direct band gap semiconductor composed entirely of group IV elements ${ }^{4}$. The tunability of its band gap makes $\mathrm{Sn}_{x} \mathrm{Ge}_{1-x}$ a highly interesting material for infrared applications, especially at low Sn concentrations $(x<0.20)^{5}$. Recent theoretical calculations indicate that strained $\mathrm{Sn}_{x} \mathrm{Ge}_{1-x}(x<0.10)$ exhibits enhanced electron and hole mobility, which could make this alloy also interesting for high speed integrated circuits ${ }^{6}$.

A number of studies have been performed to investigate the diffusion of $\mathrm{Sn}$ in germanium with secondary ion mass spectrometry ${ }^{7,8}$. More recently, using a radiotracer technique, Riihimäki et al. explained the diffusion mechanism of $\mathrm{Sn}$ in Ge by assuming an attractive elastic interaction between the $\mathrm{Sn}$ atoms and vacancies. They demonstrated that Sn diffusion is mediated by vacancies, and that the Sn-vacancy complex is negatively charged in $\mathrm{Ge}^{9,10}$. Although it is clear that $\mathrm{Sn}$ diffusion is mediated by vacancies, little is known about the microscopic configuration of Sn atoms in Ge, in particular when they are part of small defect complexes, such as the Sn-vacancy complex. It is generally accepted that the majority (if not all) of the Sn atoms is located substitutionally in a Ge matrix due to its isovalency. So far, most of the experimental information about the lattice site location of $\mathrm{Sn}$ and Sn-related defects in Ge is obtained from Mössbauer spectroscopy (MS) and Rutherford backscattering spectrometry in channeling geometry (RBS/C). Taraci et al. used RBS/C experiments to investigate the lattice location of $\mathrm{Sn}$ in $\mathrm{Sn}_{x} \mathrm{Ge}_{1-x}$ layers with different concentrations $(x=0.02,0.06$ and 0.12$)$, resulting only in the observation of Sn atoms on the substitutional site ${ }^{11}$. By combining MS and RBS/C experiments, Weyer et al. found that $90 \%$ of the ${ }^{119 m}$ Sn atoms were located substitutionally after implantation at 450 ${ }^{\circ} \mathrm{C}^{12}$. However, after implantation of ${ }^{119} \mathrm{Cd},{ }^{119} \mathrm{In},{ }^{119} \mathrm{Sb}$ and ${ }^{119 \mathrm{~m}} \mathrm{Te}$ as precursors for Sn, the Mössbauer spectra for Sn were found to consist of several contributions (resonances) with the most pronounced line interpreted as substitutional Sn and the others as several other configurations, such as isolated tetrahedral interstitial Sn atoms and two different Sn-vacancy complexes ${ }^{13-15}$. Although in MS experiments the Sn atoms may inherit the geometrical structure of the defects of the parents isotopes, it is still possible to measure the hyperfine parameters of the Sn atoms on different lattice sites and in several defect complexes. However, it should be pointed out that from MS alone, it is not possible to unambiguously determine the microscopic configuration of such defect complexes.

The situation is different in theoretical work, where the atomic arrangement in a specific complex, e.g. the Sn-vacancy complex, can be imposed. The two most simple configurations for such an impurity-vacancy complex have been studied with density functional theory ${ }^{16}$ : (a) the so-called full-vacancy configuration in which the impurity is located on a substitutional site with a vacancy as nearest neighbor, and (b) the split-vacancy configuration where the impurity atom occupies the bondcentered (BC) site in between two vacant nearest neigh- 
(a)

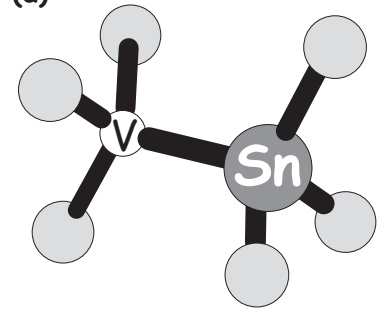

(b)

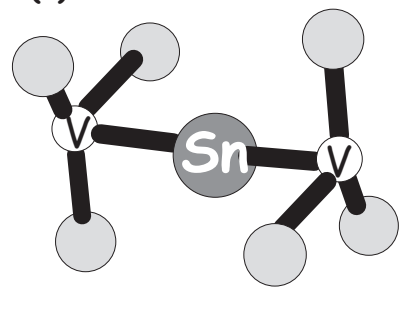

proposed by Coutinho et al. ${ }^{17}$, in order to validate our experimental results. Furthermore, these calculations will allow us to compare the deduced hyperfine parameters (isomer shift and quadrupole splitting) of the Sn atoms to the experimental values obtained from Mössbauer spectroscopy experiments ${ }^{13-15}$.

\section{EXPERIMENTAL METHOD}

bor sites. These configurations are schematically represented in Fig. 1 (a) and (b) respectively.

Höhler et al. found that the split-vacancy configuration is energetically the most favorable one for the high- $\mathrm{Z}$ elements $\mathrm{Cd}$, In, Sn, Sb and $\mathrm{Bi}$, while the full-vacancy configuration is preferred for the lower-Z elements $\mathrm{Al}$, $\mathrm{Si}, \mathrm{P}, \mathrm{Ga}, \mathrm{As}$, and $\mathrm{Se}^{16}$. One year later, comparable calculations were performed by Coutinho et al., where all studied donor-vacancy complexes (P, As, Sb and $\mathrm{Bi}$ ) were calculated to be in the full-vacancy configuration ${ }^{17}$. According to Coutinho et al., the contradicting results between the two groups for the high-Z elements $\mathrm{Sb}$ and $\mathrm{Bi}$ can be attributed to the smaller size of the supercell and the different $k$-point sampling method used by Höhler et al.

Hence, it is clear from literature that experimental information about the microscopic structure of Sn-vacancy complexes in Ge is largely lacking, while the theoretical results obtained by Höhler et al. have been put into question. Although electron paramagnetic resonance experiments in silicon have indicated that the Sn atom occupies the bond-centered site in a Sn-vacancy complex ${ }^{18}$, no unambiguous experimental evidence has been provided concerning the microscopic configuration of the Sn-vacancy complex in germanium.

In this study, we have determined the exact lattice location of ion implanted Sn atoms, both experimentally using the emission channeling technique - and theoretically. The use of ion implantation assures that a large number of vacancies, which are known to be mobile at room temperature ${ }^{19}$, are present, enhancing the probability to create Sn-vacancy complexes. Recent emission channeling experiments with the optical dopant Er, the electrical dopant In and with the transition metals $\mathrm{Fe}, \mathrm{Cu}$ and $\mathrm{Ag}$ in germanium, have proven that this technique is very effective in determining the lattice location of impurities and impurity-related defects in a direct way ${ }^{20-22}$. Besides emission channeling experiments, we have also performed theoretical calculations to determine the heat of formation and the hyperfine parameters of the relaxed configurations with Sn atoms on several high-symmetry sites and in several simple defect complexes in Ge. These calculations are performed using a different $k$-point sampling method and a larger supercell than in Ref. 16, as

Emission channeling (EC) makes use of the fact that charged particles (in this case electrons), emitted from implanted radioactive isotopes, are guided by the potential of atomic rows and planes while traveling through a single crystal. The resulting anisotropic electron emission patterns around low-index crystal directions are characteristic for the lattice site occupied by the emitting atom and are measured with a two-dimensional energy- and position-sensitive Si detector of $22 \times 22$ pixels. Applying this technique, it becomes possible to measure the lattice location of impurities with a very high accuracy $(<0.2 \AA)$ and with a sensitivity which is typically more than one order of magnitude higher than ion channeling spectroscopy (RBS/C). More information about the technique can be found in the article by Wahl et $a l^{23}$. To obtain unambiguous quantitative results of the lattice location of the impurity under investigation, the electron emission patterns are measured around four independent crystal directions $(\langle 100\rangle,\langle 111\rangle,\langle 211\rangle$ and $\langle 110\rangle)$, analyzed consistently and fitted to a set of simulated patterns. These simulations ${ }^{24}$ are based on the dynamical theory of electron diffraction, and are calculated for the substitutional (S), tetrahedral interstitial (T), bondcentered (BC), and the so-called SP, H, Y and C sites ${ }^{25}$, together with discrete displacements between all of these high-symmetry sites along the $\langle 111\rangle$ - and $\langle 100\rangle$-direction.

To study the lattice location of Sn atoms in a Ge matrix, we implanted the radioactive isotope ${ }^{121} \mathrm{Sn}$ which decays to ${ }^{121} \mathrm{Sb}$ with a half life of $27 \mathrm{~h}$, emitting $\beta$ electrons with an endpoint energy of $390 \mathrm{keV}$. The implantations were performed at the ISOLDE facility in CERN at room temperature, in undoped $\langle 111\rangle-\mathrm{Ge}$, with an energy of $60 \mathrm{keV}$ and at an offset angle of $7^{\circ}$ with respect to the surface normal to minimize channeled implantation. Three slightly different implantation fluences have been used: $2 \times 10^{12}, 3 \times 10^{12}$ and $4 \times 10^{12}$ Snatoms $/ \mathrm{cm}^{2}$. The current density used during implantation was $0.3-0.4 \mathrm{nA} / \mathrm{cm}^{2}$. The measurements have been performed at room temperature, and to monitor the thermal stability of the lattice location of the implanted species, spectra were taken directly after implantation as well as after $10 \mathrm{~min}$ annealing stages in vacuum $\left(<10^{-5}\right.$ mbar) at temperatures ranging up to $400{ }^{\circ} \mathrm{C}$. 


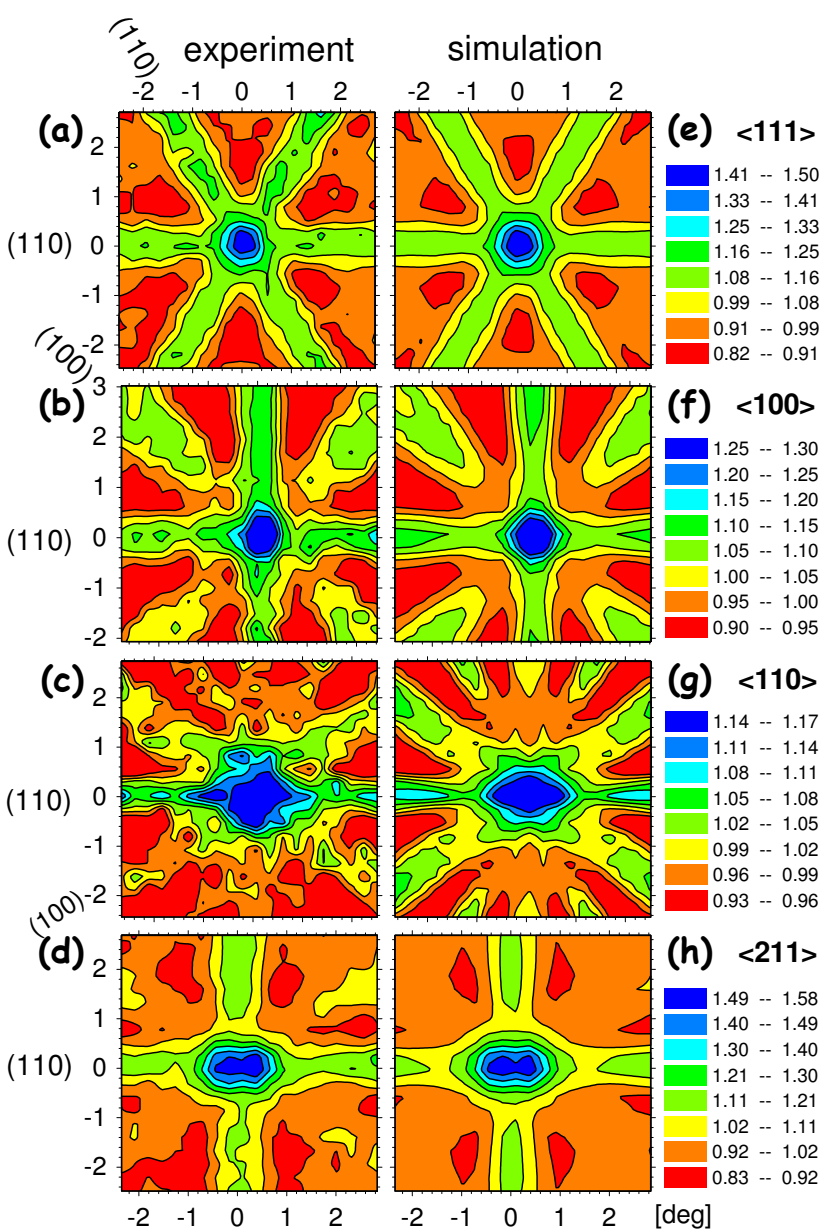

FIG. 2: (Color online) (a)-(d) Two-dimensional normalized electron emission patterns from ion implanted ${ }^{121} \mathrm{Sn}$ in Ge to a fluence of $3 \times 10^{12}$ atoms $/ \mathrm{cm}^{2}$, measured around the $\langle 111\rangle$, $\langle 100\rangle,\langle 110\rangle$ and $\langle 211\rangle$-axis respectively, following a $300{ }^{\circ} \mathrm{C}$ annealing in vacuum; (e)-(h) The best fits to these patterns, with the majority (on average $76 \%$ ) of ${ }^{121} \mathrm{Sn}$ atoms occupying the substitutional site and a smaller fraction (on average 11\%) on the bond-centered site.

\section{EXPERIMENTAL RESULTS}

Figs. 2(a) -2 (d) show the measured electron emission patterns from the implanted ${ }^{121} \mathrm{Sn}$ atoms in $\mathrm{Ge}\left(3 \times 10^{12}\right.$ atoms $\left./ \mathrm{cm}^{2}\right)$ around the $\langle 111\rangle,\langle 100\rangle,\langle 110\rangle$ and $\langle 211\rangle$-axis respectively, following a $300{ }^{\circ} \mathrm{C}$ annealing. All patterns are dominated by pronounced channeling effects, indicating that the majority of the probe atoms are located along the rows of atoms in the measured directions, i.e. on the substitutional (S) site. To investigate small contributions of other high-symmetry sites and to obtain accurate quantitative information about the fractional occupation of the lattice sites, the experimental patterns have been fit by simulated patterns. Up to three different highsymmetry sites were used in the fitting procedure, including small displacements of these sites as well. The only high-symmetry site that, when added to the sub- stitutional fraction, results in a significantly improved fit which is consistent in all four measured directions (a reduced $\chi^{2}$-improvement of more than $4 \%$ ), is the bondcentered (BC) site. Adding a third high-symmetry site did not further improve the fit ( $\chi^{2}$-improvement $\left.\ll 1 \%\right)$. Using slightly displaced S and/or BC sites only results in very small fit improvements, which is most likely due to the extra degrees of freedom in the fitting procedure. In Figs. 2(e) $-2(\mathrm{~h})$, the best fits to the experimental patterns are shown, using undisplaced $\mathrm{S}$ and $\mathrm{BC}$ sites. The fractions used to produce these fits are $78 \%, 79 \%, 70 \%$ and $75 \%$ on the S site and $11 \%, 9 \%, 14 \%$ and $11 \%$ on the BC site for the $\langle 111\rangle,\langle 100\rangle,\langle 110\rangle$ and $\langle 211\rangle$-direction respectively, which indicates the good consistency in the fits for the four measured directions.

As can be seen in Fig. 3, similar results are obtained for the three investigated samples, directly after implantation as well as after several annealing stages. The majority of the $\mathrm{Sn}$ atoms is located substitutionally $\left(f_{\mathrm{S}}\right)$, with a smaller but significant fraction occupying the BC site $\left(f_{\mathrm{BC}}\right)$. It is important to note the thermal stability of the Sn atoms on the bond-centered site as well as on the substitutional site, up to at least $400{ }^{\circ} \mathrm{C}$. The remaining fraction of the Sn atoms, $f_{\text {random }}=100 \%-f_{\mathrm{S}}-f_{\mathrm{BC}}$ is the so-called random fraction and has been added to Fig. 3 for clarity. This random fraction represents the Sn atoms that are not located on high-symmetry sites, only adding up to the homogeneous background of the pattern.

The results in Fig. 3 show no direct influence of the implantation fluence on the lattice location behavior of the Sn atoms, within the range of $2-4 \times 10^{12}$ atoms $/ \mathrm{cm}^{2}$. It is clear that directly after ion implantation, a relatively high random fraction is present. This is a direct consequence of the implantation-induced lattice damage, which has a dual influence on the results shown here. Firstly, due to the deterioration of the crystal structure, a fraction of the implanted radioactive isotopes will be located in damaged regions with reduced local crystallinity. Secondly, a fraction of the electrons emitted from an undamaged region will pass through damaged crystal regions, enhancing the probability for dechanneling - thus masking the impurity's lattice site. Both effects will result in an isotropic background to the patterns and consequently in the random fraction, as observed in the experiments. Therefore, the presented fractional occupation of the Sn atoms on the high-symmetry sites for the lower temperature annealing steps $\left(<300{ }^{\circ} \mathrm{C}\right)$ must be regarded as a lower limit to the real values. The recovery of the lattice damage after annealing is reflected in the large decrease of the random fraction after annealing the samples at $300{ }^{\circ} \mathrm{C}$, resulting in almost all $\mathrm{Sn}$ atoms on high-symmetry sites. 


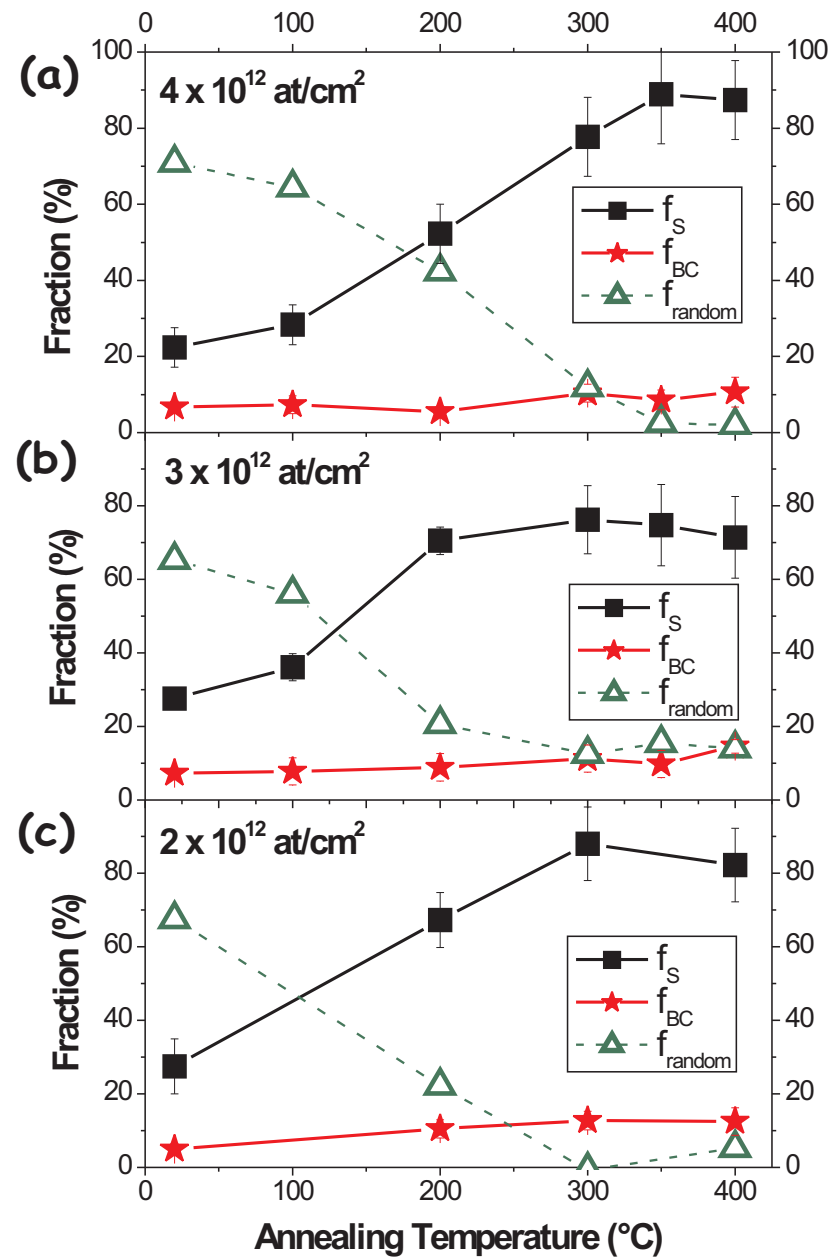

FIG. 3: (Color online) Fraction of the implanted Sn atoms on the substitutional (squares) and bond-centered site (stars) in $\mathrm{Ge}$, together with the random fraction $\mathrm{f}_{\text {random }}=100 \%$ - $f_{S}-f_{B C}$ (triangles) for three slightly different implantation fluences: (a) $4 \times 10^{12}$, (b) $3 \times 10^{12}$ and (c) $2 \times 10^{12}$ atoms $/ \mathrm{cm}^{2}$.

\section{CALCULATIONS}

As expected, the majority of the isovalent $\mathrm{Sn}$ atoms are located substitutionally in the Ge lattice. Most likely, many of these impurities are embedded in an undamaged environment, although it cannot be excluded that some of them are surrounded by simple defects, without influencing their substitutional position. A smaller but significant fraction of $\mathrm{Sn}$ atoms is found to occupy the $\mathrm{BC}$ site, which has not been reported for Sn atoms in Ge so far. As mentioned above, this BC fraction is possibly related to the Sn-vacancy defect in the split-vacancy configuration, as presented in Fig. 1(b). To strengthen this assumption, a complementary ab initio study has been performed.

We have calculated the heat of formation of several impurity sites for $\mathrm{Sn}$ in Ge: the S site (with and without a vacancy or a Ge self-interstitial as nearest neighbor), the $\mathrm{T}$ site (with and without a vacancy as nearest neighbor) and the BC site (in the split-vacancy configuration and without any vacancies nearby). All defect complexes were allowed to completely relax to their most stable configuration. The heats of formation reported in Tab. I are calculated according to

$$
\Delta H_{f}=E_{\text {sup }}^{i m p}-\mu_{i m p}-\left(32 E_{\text {sup }}^{i d}-n \mu_{G e}\right)
$$

where $E_{\text {sup }}^{i m p}$ is the total energy of a 63- or 64-atom supercell that contains the impurity, $E_{\text {sup }}^{\text {id }}$ is the total energy of a pure Ge unit cell (diamond structure, 2 atoms), $\mu_{G e}$ is the chemical potential of Ge (taken equal to the total energy per atom in bulk Ge), $n$ is the number of Ge atoms in the ideal 64-atom supercell that are replaced by either vacancies or impurities $(n=1,2)$ and $\mu_{i m p}$ is the chemical potential of Sn (taken equal to the total energy per atom in bulk $\alpha$-Sn). The Ge lattice constant was optimized and fixed for the 64-atom cells. The calculations were done by the APW+lo method ${ }^{26}$ within Density Functional Theory ${ }^{27}$, as implemented in the WIEN2k code ${ }^{28,29}$. The Perdew-Burke-Ernzerhof ${ }^{30}$ exchange-correlation functional was used, the $k$-space sampling was done on a $4 \times 4 \times 4$ mesh in the 64 -atom cell, and a basis set corresponding to $K_{\max }=3.5$ a.u. was taken. The influence of the size of the supercell (up to 256 atoms) on the calculations was verified and found to be negligible. In this way, we have performed calculations in a similar way as Höhler et $a l^{16}$, while also taking into account the suggestions of Coutinho et al. ${ }^{17}$, i.e. increasing the number of $k$-points and the size of the supercell.

After relaxation of the configuration with the Sn impurity on the $\mathrm{S}$ site $\left(\mathrm{Sn}_{\mathrm{S}}\right)$ and the $\mathrm{T}$ site $\left(\mathrm{Sn}_{\mathrm{T}}\right)$, we found a local expansion of the nearest neighbor distance of $5 \%$ and $8 \%$ respectively. When adding a vacancy as a nearest neighbor to the substitutional impurity, the calculations indicate a large force on the Sn atom along the $\langle 111\rangle$-direction of the Ge crystal, resulting in the relocation of the Sn impurity to the 6-fold coordinated BC site with the vacancy split over the nearest neighbor positions: the split-vacancy configuration (see Fig. 1(b)). Although having a different $k$-point sampling method and an increased supercell size, these results are in agreement with what was found by Höhler et $a l^{16}$ : the $\mathrm{Sn}_{\mathrm{S}}$-vacancy complex in the full-vacancy configuration is not stable in Ge and spontaneously relaxes to the split-vacancy configuration.

When adding a nearest neighbor vacancy to the $\mathrm{Sn}_{\mathrm{T}}$ configuration, the interstitial $\mathrm{Sn}$ atom feels a force towards the vacant site. As a result, this configuration is not stable either, resulting in the relocation of the Sn atom to the substitutional site. When relaxing the configuration with $\mathrm{Sn}$ on the BC site and no vacancies nearby, no lattice site changes (but relatively large displacements of the surrounding Ge atoms) were observed. Finally, the configuration with a Ge self-interstitial as nearest neighbor of a substitutional Sn impurity was found to be stable, i.e. no lattice site changes were observed. 


\begin{tabular}{lcccccc}
\hline \hline & $\begin{array}{c}\Delta H_{f} \\
(\mathrm{eV})\end{array}$ & $\begin{array}{c}\delta_{0(\text { calc })} \\
(\mathrm{mm} / \mathrm{s})\end{array}$ & $\begin{array}{c}\Delta \mathrm{E}_{Q(\text { calc })} \\
(\mathrm{mm} / \mathrm{s})\end{array}$ & $\begin{array}{c}\mathrm{MB} \\
\text { line } \mathrm{nr}^{13-15}\end{array}$ & $\begin{array}{c}\delta_{(\exp )} \\
(\mathrm{mm} / \mathrm{s})\end{array}$ & $\begin{array}{c}\Delta \mathrm{E}_{Q(\exp )} \\
(\mathrm{mm} / \mathrm{s})\end{array}$ \\
\hline $\mathrm{Sn}_{\mathrm{S}}$ & 0.19 & 1.75 & 0.0 & 2 & 1.90 & 0.0 \\
$\mathrm{Sn}_{\mathrm{T}}$ & 3.96 & 3.19 & 0.0 & 4 & 3.27 & 0.0 \\
$\mathrm{Sn}_{\mathrm{BC}}$ (split-vacancy) & 1.86 & 2.24 & 0.10 & 3 & 2.36 & $0.3^{13}-0.4^{14,15}$ \\
$\mathrm{Sn}_{\mathrm{BC}}$ (no vacancies) & 3.83 & 3.25 & 0.82 & & & \\
$\mathrm{Sn}_{\mathrm{S}}+\mathrm{Ge}_{\mathrm{T}}$ (self-int.) & 3.51 & 1.84 & 0.64 & & & \\
unknown & & & & 1 & 1.41 & 0.0 \\
\hline \hline
\end{tabular}

TABLE I: Heats of formation $\Delta H_{f}(\mathrm{eV})$, calculated isomer shift $\delta_{0(\text { calc })}(\mathrm{mm} / \mathrm{s})$ and quadrupole splitting $\Delta \mathrm{E}_{Q(\text { calc })}(\mathrm{mm} / \mathrm{s})$ for the relaxed impurity environments considered in this work: Sn on the $\mathrm{S}$ site $\left(\mathrm{Sn}_{\mathrm{S}}\right)$, on the T site $\left(\mathrm{Sn}_{\mathrm{T}}\right)$, on the BC site in the split-vacancy configuration $\left(\mathrm{Sn}_{\mathrm{BC}}\right.$ (split-vacancy)), $\mathrm{Sn}$ on the $\mathrm{BC}$ site without vacancies nearby ( $\mathrm{Sn}_{\mathrm{BC}}($ no vacancies)), and substitutional $\mathrm{Sn}$ with a self-interstitial as nearest neighbor $\left(\mathrm{Sn}_{\mathrm{S}}+\mathrm{Ge}_{\mathrm{T}}\right.$ (self-int.)). A comparison is made to the experimental isomer shift $\delta_{0(\exp )}(\mathrm{mm} / \mathrm{s})$ and the quadrupole splitting $\Delta \mathrm{E}_{Q(\exp )}(\mathrm{mm} / \mathrm{s})$ as deduced from the 4 Mössbauer spectroscopy lines in Refs. 13, 14 and 15.

\section{DISCUSSION}

By comparing the heats of formation in Tab. I, it is clear that the Sn atoms prefer the S site to the other high-symmetry sites. This is consistent with previous experiments where the majority of Sn atoms were found on the $\mathrm{S}$ site ${ }^{11,12}$, with our emission channeling results and with what one would expect for isovalent impurities. Although the heats of formation in Tab. I suggest that Sn on the $\mathrm{BC}$ site is not energetically favorable with respect to substitutional Sn, the following arguments show that this is not the case. We calculated the heat of formation for a single neutral vacancy in Ge to be $2.23 \mathrm{eV}$ (in agreement with literature $\left.{ }^{31}\right)$. The total energy needed to have an impurity on the $\mathrm{S}$ site and an isolated vacancy not bound to it $(0.19 \mathrm{eV}+2.23 \mathrm{eV}=2.42 \mathrm{eV})$ is significantly larger than the heat of formation of the split-vacancy configuration of the Sn-vacancy complex $(1.86 \mathrm{eV})$. This result is in agreement with the attractive interaction between $\mathrm{Sn}$ atoms and vacancies found by Riihimäki et al. ${ }^{9}$. Therefore, one can conclude that a substitutional Sn atom will likely trap one of the abundantly available mobile vacancies created during implantation, and - as the structural relaxation process in our calculations shows - will spontaneously evolve into the BC site. Consequently, our experimental results are well corroborated by the presented $a b$ initio calculations.

Additionally, in order to allow comparison of our results with the results from the above mentioned Mössbauer spectroscopy experiments ${ }^{13-15}$, we have calculated the isomer shift (relative to $\mathrm{CaSnO}_{3}$ ) and the quadrupole splitting for ${ }^{119} \mathrm{Sn}$ atoms on several highsymmetry lattice sites, with and without a vacancy or a self-interstitial nearby. For each configuration, we investigated the influence of the magnetic moment $(0,1$ and 2 $\left.\mu_{B}\right)$ and the charge state $(2+, 1+$ and $0+)$ of the impurity atom on the calculated hyperfine parameters. The calculated values (open symbols) and experimental values from previous MS experiments (closed symbols) are visually presented in Fig. 4 and are also listed in Tab. I for clarity. As can be seen from Fig. 4, the charge state and the magnetic moment of the impurity atom do not

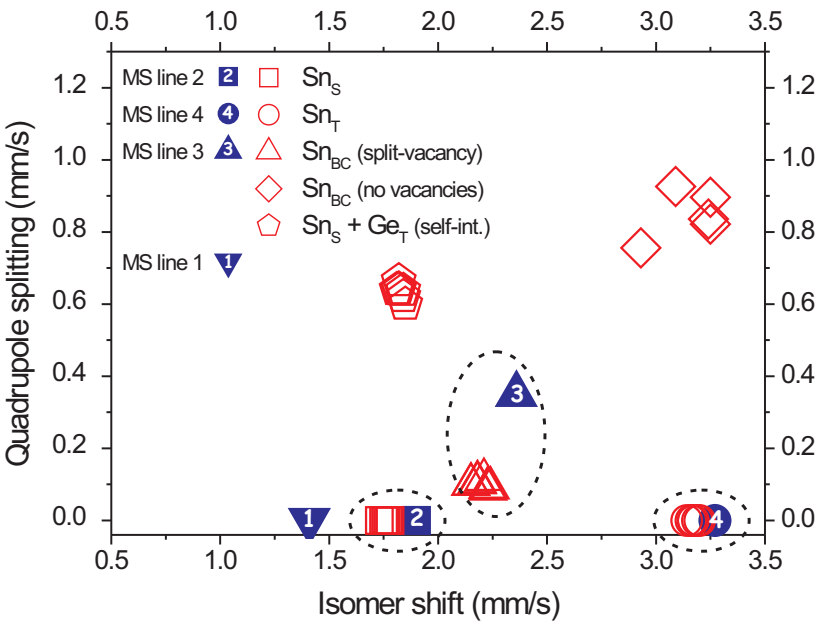

FIG. 4: (Color online) Calculated (open symbols) and measured ${ }^{13-15}$ (closed numbered symbols) hyperfine parameters (quadrupole splitting versus isomer shift) of Sn impurities on the substitutional site $\left(\mathrm{Sn}_{\mathrm{S}}\right.$ - squares), on the tetrahedral interstitial site $\left(\mathrm{Sn}_{\mathrm{T}}\right.$ - circles), on the bond-centered site in the split-vacancy configuration ( $\mathrm{Sn}_{\mathrm{BC}}$ (split-vacancy) - triangles up), on the bond-centered site without vacancies nearby ( $\mathrm{Sn}_{\mathrm{BC}}$ (no vacancies) - diamonds) and on the substitutional site with a self-interstitial as nearest neighbor $\left(\mathrm{Sn}_{\mathrm{S}}+\mathrm{Ge}_{\mathrm{T}}\right.$ (self-int.) - pentagons). For each configuration, the calculations have been performed for different charge states and magnetic moments.

have a significant influence on the calculated hyperfine parameters, and therefore will not be discussed in more detail.

In Refs. 13, 14 and 15, four different Mössbauer resonances (labeled as line 1, 2, 3 and 4) were identified. Lines 2 and 4 were assigned to Sn atoms in a tetrahedral symmetrical surrounding, i.e. on the $\mathrm{S}$ and the $\mathrm{T}$ site, respectively. These assignments are in good agreement with our calculated hyperfine parameters for these sites, as can be seen from Tab. I and Fig. 4 . Line 3 and line 1 were interpreted as Sn-vacancy complexes in the fullvacancy and the split-vacancy configuration, respectively. 
Our calculations show that the hyperfine parameters of the bond-centered $\mathrm{Sn}$ atoms in the split-vacancy configuration are not in agreement with line 1 , but in relatively good agreement ${ }^{32}$ with line 3 , indicating that the tentative assignment of these two Mössbauer lines was not correct. These results show that the Sn-vacancy complex in the split-vacancy configuration has been measured indirectly before, however without having been identified as such. Since the hyperfine parameters of the other calculated configurations are not in agreement with the experimental values of line 1 , it has not been possible to assign a specific configuration to this Mössbauer resonance. As a general quality check, we note that the isomer shift values calculated by Höhler - i.e. $1.88 \mathrm{~mm} / \mathrm{s}$ for substitutional $\mathrm{Sn}$ and $2.16-2.19 \mathrm{~mm} / \mathrm{s}$ for $\mathrm{Sn}$ in the split-vacancy configuration $^{16}$ - are in good agreement with our calculations.

\section{CONCLUSIONS}

In conclusion, we have performed a lattice location study of ion implanted Sn in Ge. The majority of the isovalent Sn impurities are located substitutionally (S), although a significant fraction $(\sim 15 \%)$ of the Sn atoms was found on the bond-centered (BC) site. Both sites are thermally stable up to at least $400{ }^{\circ} \mathrm{C}$. Backed up by $a b$ initio density functional calculations, we have been able to relate the bond-centered Sn atoms with Sn-vacancy complexes in the split-vacancy configuration. Moreover, calculations of the hyperfine parameters of this defect indicate that it has probably been present in Mössbauer experiments before, without having been identified as such. In our work, we have presented strong evidence, both experimentally and theoretically, that the Sn-vacancy de- fect prefers the split-vacancy configuration which is thermally stable up to $400{ }^{\circ} \mathrm{C}$. This result is of major importance in the understanding of the problems that arise in the formation of diluted $\mathrm{Sn}_{\mathrm{x}} \mathrm{Ge}_{1-\mathrm{x}}$ alloys, since recent calculations have indicated that the split-vacancy configuration of the $\mathrm{Sn}$ atom in $\mathrm{Ge}$ is a nucleation point of metallic $\mathrm{Sn}^{33}$, hence deteriorating the semiconducting properties. Besides this, our results contribute to the study of simple point defects in elemental group IV semiconductors and are important to understand the vacancymediated mechanism of Sn diffusion - and maybe even self-diffusion - in germanium.

The transition metals $\mathrm{Fe}, \mathrm{Cu}$ and $\mathrm{Ag}$, and the acceptor In were experimentally observed and calculated to be on the $\mathrm{BC}$ site in the impurity-vacancy complex in Ge as well ${ }^{16,21,22}$. Therefore, a general trend can be expected for the transition metals, group III and group IV impurities in Ge. However, preliminary results of similar calculations for group $\mathrm{V}$ elements indicate that this trend can not be generalized for all impurities in $\mathrm{Ge}^{34}$, highlighting the need of additional experimental and theoretical studies on impurity-vacancy complexes in Ge.

\section{Acknowledgments}

This work was supported by the Fund for Scientific Research, Flanders (FWO G.0501.07 and G.0636.08), the Concerted Action of the K.U.Leuven (GOA/2009/006), the Inter-University Attraction Pole (IUAP P6/42), the Center of Excellence Programme (INPAC EF/2005/005), the Portuguese Foundation for Science and Technology (CERN-FP-83506-2008) and the ISOLDE collaboration. S.D. acknowledges his fellowship from FWO, Flanders and from the Research Council of the K.U.Leuven.
Electronic address: stefan.decoster@fys.kuleuven.be

1 R. Hull and J. C. Bean, Germanium Silicon: Physics and Materials, Semiconductors and Semimetals, Academic, San Diego (1999).

2 C. C. Yeo, B. J. Cho, F. Gao, S. J. Lee, M. H. Lee, C. Y. Yu, C. W. Liu, L. J. Tang, and T. W. Lee, IEEE Electron Dev. Lett. 26, 761 (2005).

3 Y. J. Yang, W. S. Ho, C. F. Huang, S. T. Chang, and C. W. Liu, Appl. Phys. Lett. 91, 102103 (2007).

4 C. H. L. Goodman, IEE Proc., Part I: Solid-State Electron Devices 129, 189 (1982).

${ }^{5}$ G. He and H. A. Atwater, Phys. Rev. Lett. 79, 1937 (1997).

6 J. D. Sau and M. L. Cohen, Phys. Rev. B 75, 045208 (2007).

7 P. Kringhøj and R. G. Elliman, Appl. Phys. Lett. 65, 324 (1994).

8 M. Friesel, U. Sodervall, and W. Gust, J. Appl. Phys. 78, 5351 (1995).

9 I. Riihimäki, A. Virtanen, H. Kettunen, P. Pusa, J. Räisänen, and the ISOLDE Collaboration, Appl. Phys. Lett. 90, 181922 (2007).
10 I. Riihimäki, A. Virtanen, S. Rinta-Anttila, P. Pusa, J. Räisänen, and the ISOLDE Collaboration, Appl. Phys. Lett. 91, 091922 (2007).

11 J. Taraci, S. Zollner, M. R. McCartney, J. Menendez, M. A. Santana-Aranda, D. J. Smith, A. Haaland, A. V. Tutukin, G. Gundersen, G. Wolf, et al., J. Am. Chem. Soc. 123, 10980 (2001).

12 G. Weyer, A. Nylandsted-Larsen, B. I. Deutch, J. U. Andersen, and E. Antoncik, Hyperf. Int. 1, 93 (1975).

13 G. Weyer, S. Damgaard, J. W. Petersen, and J. Heinemeier, Phys. Lett. 76A, 321 (1980).

14 G. Weyer, J. W. Petersen, and S. Damgaard, Hyperf. Int. 10, 775 (1981).

15 S. Damgaard, A. F. F. Olesen, J. W. Petersen, and G. Weyer, Physica Scripta 22, 640 (1981).

16 H. Höhler, N. Atodiresei, K. Schroeder, R. Zeller, and P. H. Dederichs, Phys. Rev. B 71, 035212 (2005).

17 J. Coutinho, S. Öberg, V. J. B. Torres, M. Barroso, R. Jones, and P. R. Briddon, Phys. Rev. B 73, 235213 (2006).

18 G. D. Watkins, Phys. Rev. B 12, 4383 (1975). 
19 H. Haesslein, R. Sielemann, and C. Zistl, Phys. Rev. Lett. 80, 2626 (1998).

20 S. Decoster, B. De Vries, U. Wahl, J. G. Correia, and A. Vantomme, Appl. Phys. Lett. 93, 141907 (2008).

${ }^{21}$ S. Decoster, S. Cottenier, B. De Vries, H. Emmerich, U. Wahl, J. G. Correia, and A. Vantomme, Phys. Rev. Lett. 102, 065502 (2009).

22 S. Decoster, B. De Vries, U. Wahl, J. G. Correia, and A. Vantomme, J. Appl. Phys. 105, 083522 (2009).

23 U. Wahl, J. G. Correia, A. Czermak, S. G. Jahn, P. Jalocha, J. G. Marques, A. Rudge, F. Schopper, J. C. Soares, A. Vantomme, et al., Nucl. Instrum. Methods Phys. Res. A 524, 245 (2004).

${ }^{24}$ H. Hofsäss and G. Lindner, Phys. Rep. 201, 121 (1991).

25 U. Wahl, Phys. Rep. 280, 145 (1997).

${ }^{26}$ E. Sjöstedt, L. Nordström, and D. J. Singh, Solid State Commun. 114, 15 (2000).

27 P. Hohenberg and W. Kohn, Phys. Rev. 136, 864 (1964).

28 S. Cottenier, (Instituut voor Kern- en Stralingsfysica, KULeuven, Belgium) (2002), (freely available from http://www.wien2k.at/reg_user/textbooks), ISBN 90-807215-1-4.

${ }^{29}$ P. Blaha, K. Schwarz, G. Madsen, D. Kvasnicka, and
J. Luitz, (Karlheinz Schwarz, Techn. Universität Wien, Austria) (1999), ISBN 3-9501031-1-2.

30 J. P. Perdew, K. Burke, and M. Ernzerhof, Phys. Rev. Lett. 77, 3865 (1996).

31 J. Vanhellemont, P. Spiewak, and K. Sueoka, J. Appl. Phys. 101, 036103 (2007).

32 Due to the relatively small quadrupole moment $(0.132 \mathrm{~b})$ of ${ }^{119} \mathrm{Sn}$, in the MS measurements, a small electric field gradient results in an unresolved doublet, i.e. a line which is only slightly broader than the natural line width. This makes an accurate determination of the quadrupole splitting very difficult and explains the difference between the calculated and the experimentally observed quadrupole splitting. However, since the experimental value of the isomer shift, which can be determined much more accurately, is very close to the calculated value, we can still conclude that the experimental and calculated hyperfine parameters of $\mathrm{Sn}_{\mathrm{BC}}$ in the split-vacancy configuration are in good agreement.

${ }^{33}$ C. I. Ventura, J. D. Fuhr, and R. A. Barrio, Phys. Rev. B 79, 155202 (2009).

${ }^{34}$ S. Cottenier, private communication. 